\title{
Effect of the Imperfect Flat Earth on the Vertically Polarized Radiation of a Cylindrical Reflector Antenna
}

\author{
Svetlana V. Boriskina, Student Member, IEEE, Alexander I. Nosich, Senior Member, IEEE, and \\ Ayhan Altintaş, Senior Member, IEEE
}

\begin{abstract}
The radiation of a circular cylindrical reflector antenna in the presence of imperfect flat earth is treated in an accurate manner. The boundary value problem is formulated in terms of a full-wave integral equation converted to the dual-series equations and then regularized by using analytical inversion of the static part. The resulting Fredholm second-kind matrix equation is solved numerically with guaranteed accuracy. The feed directivity is included in the analysis by using the complex source-point method. Various antenna features, which include the overall directivity, efficiency, gain, and radiated and absorbed power fractions have been calculated and compared with the free-space antenna characteristics. They show some phenomena not predicted by approximate techniques.
\end{abstract}

Index Terms-Analytical regularization, complex source, cylindrical reflectors, imperfect earth.

\section{INTRODUCTION}

$\mathbf{R}$ EFLECTORS are among the oldest and most popular antenna configurations used in radar and communication applications. Their properties have been studied by various methods of analysis; among the most popular are the physical optics (PO) and the geometrical theory of diffraction (GTD). Although useful and versatile, both are high-frequency approximations that must be confronted by an exact solution for a test of accuracy. For example, without essential modifications, PO is not able to predict the side and backward radiation, while GTD fails in the main beam direction. Another commonly used approach is the method of moments (MoM), which is based on the exact electric field integral equations (EFIE) discretized by using subdomain or entire-domain expansion functions. However, EFIE is normally a first-kind integral equation and frequently has a hypersingular kernel. This prevents a proof of convergence of usual discretization schemes: the computational error cannot be progressively minimized by increasing the size of an "impedance matrix." Despite this, MoM is normally able to predict the reflector performance with a practical accuracy

Manuscript received January 20, 1998; revised October 12, 1999. The work of S. V. Boriskina was supported by the SUMMA Foundation in the form of a Graduate Fellowship in Advanced Electromagnetics.

S. V. Boriskina is with the Radiophysics Department, Kharkov State University, Kharkov, 310077 Ukraine.

A. I. Nosich is with the Institute of Radiophysics and Electronics, National Academy of Sciences, Kharkov, 310085 Ukraine . He is also with the Laboratoire Antennes et Reseaux, Universite de Rennes 1, Rennes, 35042 France.

A. Altintaş is with the Department of Electrical and Electronics Engineering, Bilkent University, Ankara, 06533 Turkey.

Publisher Item Identifier S 0018-926X(00)01270-9. provided that no sharp resonances are met, and 10-20 mesh points per wavelength $\lambda$ are used. The latter fact limits the size of computable reflectors to $20 \lambda$ (or even smaller) if antenna is located in the presence of the other scatterers.

In this paper, we use a technique that guarantees convergence and can serve as an adequate simulation tool in the quick desktop analysis of reflector antennas. Such a technique belongs to the family of those collectively called the method of analytical regularization (MAR) and was reviewed in [1]. It implies a conversion of originally singular integral equation of the first kind to the Fredholm second-kind integral equation with a smooth kernel and eventually to the equivalent Fredholm second-kind infinite-matrix equation. Then, as it was discussed in [1], the convergence is guaranteed, in the sense that computational error can be progressively minimized to the machine precision by solving greater matrices. In our case, MAR solution is based on the analytical inversion of the static part of EFIE. For a two-dimensional (2-D) circular-cylindrical perfectly electrical conducting (PEC) reflector, this was performed in detail in [2] and for a three-dimensional (3-D) spherical PEC reflector in [3]. In both analyses, MAR was combined with the complex source-point (CSP) method [4], [5] to simulate a directive primary field by a function which is an exact solution to the Helmholtz equation. As discussed in [1], MAR is equivalent to a judicious choice of the basis functions in a Galerkin-type MoM: they must form a set of orthogonal eigenfunctions of the singular part of the full EFIE operator. By using MAR, the surface currents, far-field patterns, directivity, and radiated power can be studied with a uniform and controlled accuracy (see [1]-[3]) as well as small central processing unit (CPU) time. Advantages of this MAR-CSP approach are even more apparent if the reflector is placed in a complicated near-zone environment. In [6], a cylindrical PEC reflector with nonuniform resistive edge loading was studied; it was shown that such a loading lowers the sidelobe level only in the forward half-space at the expense of a greater rear-side radiation. In [7], a similar 2-D PEC reflector, which was placed inside a circular dielectric radome, was optimized to have a maximum directivity; the latter can exceed the free-space value due to exploiting the radome as a dielectric lens. The purpose of the present paper is to extend MAR-CSP analysis to the cylindrical PEC-reflector antenna located near the imperfect flat earth. Beginning from Sommerfeld almost a century ago, earth effects have been studied, but only for the simplest antennas such as dipoles and loops [8], [9]. Our analysis deals 
with the antenna as a whole system and takes into account all the interactions, scattering and resonances, although the CSP model neglects the feed-blockage effect, and the 2-D reflector model eliminates the cross polarization.

Keeping in mind applications such as radars and radio communication, we shall be interested in obtaining the field in air. The electromagnetic properties of the earth can be taken into account by using the complex-valued surface impedance $Z$, which sets the relationship between the tangential components of the electrical and magnetic fields in the air just at the interface. Basic ideas have been reviewed in [10] for obtaining a MAR solution to the problem of the wave scattering from a cylindrical screen in a layered medium. As we are interested in the vertically polarized radiation, first the magnetic-type scalar Green's function of the half-space bounded by the impedance plane is derived in Section II. Then a hypersingular EFIE is obtained and converted to a Fredholm second-kind infinite-matrix equation by MAR. In Section III, we present the results of numerical analysis that show the far-field pattern distortions due to the earth near the reflector, the role of the earth in the gain or loss of the overall directivity and make a comparison of the influence of dry sand, wet earth, fresh water, and sea water. The time convention adopted is $\exp (-i \omega t)$, where $\omega$ is the angular frequency.

\section{PROBlem Formulation AND SOLUTION}

\section{A. Antenna Geometry and Feed Model}

The 2-D cylindrical reflector antenna presented in Fig. 1 is considered. A zero-thickness PEC reflector is located in the air above the plane surface with impedance $Z_{0} Z$, where $Z_{0}$ is the free-space impedance. The reflector cross section $M$ is assumed a circular arc of the radius $a$ elevated at the height $c$; its angular width is $2 \theta_{a p}$ and inclination angle with respect to the $x$-axis is $\varphi_{0}$. Although practical reflectors are of parabolic shape, they can be well approximated by the circular ones if the focal distance of the parabolic arc $F$ is large enough with respect to the reflector aperture $L$ (see Fig. 2 of [2] for details).

We simulate the radiation of a primary 2-D feed by using the CSP method. It means that we consider a line feed with complex source coordinates. Such a feed produces a beam field in the real space, and the greater the imaginary part of the source coordinate the narrower the radiated beam [4], [5]. This field is an exact solution of the Helmholtz equation at any observation point, unlike the Gaussian-type exponents frequently used to approximate the beam fields in paraxial domains. Thus, the complex source position vector is

$$
\vec{r}_{c s}=\left(x_{c s}, y_{c s}\right)=\vec{r}_{0}+i \vec{b}=\left(x_{0}, y_{0}\right)+(b \cos \beta, b \sin \beta) .
$$

Here, the real value $b$ is a measure of the beamwidth and the aiming angle $\beta$ measured from the $x$-axis represents the beam direction. Note that instead of a singular point, the CSP field has an associated branch cut of the length $b$ in real space (see [3] for details). Although the real part of the source coordinate $\vec{r}_{0}$ can be an arbitrary number, in the further computed examples

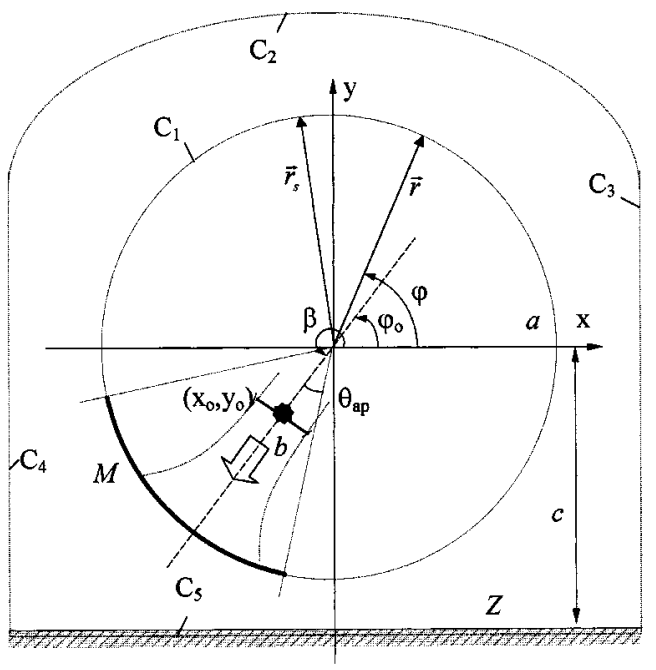

Fig. 1. Two-dimensional reflector antenna over impedance earth.

we shall assume that it coincides with the geometrical focus of reflector, i.e., is located at the distance $a / 2$ from the latter. This is because our primary goal is to study the effect of the earth on the antenna radiation. By the same reason, we shall take the feed aiming angle as $\beta=\varphi_{0}+\pi$ to ensure a symmetric feeding.

\section{B. Derivation and Discretization of the Integral Equation}

For the vertically polarized antenna radiation, the electromagnetic field can be characterized by the single $H_{z}$ component. Decomposing the total field to the sum of the incident and scattered ones, we take the former field as the one in the absence of the reflector

$$
\begin{aligned}
H^{\text {in }}(\vec{r})= & H_{0}^{(1)}\left(k\left|\vec{r}-\vec{r}_{c s}\right|\right)+\frac{1}{\pi} \int_{\infty}^{\infty} \frac{i g-\alpha}{g(i g+\alpha)} \\
& \cdot e^{i g k\left(y+y_{c s}+2 c\right)+i h k\left(x-x_{c s}\right)} d h
\end{aligned}
$$

where $k=\omega / c$ (here, $c$ is the free-space velocity of light), $\alpha=i Z, g=\sqrt{1-h^{2}}$, and $H_{0}^{(1)}$ denotes the Hankel function of order zero. The first term in (2) is due to a "magnetic-type" CSP field in free-space [1], [4]. The second one (the Fourier integral) is easily obtained by the separation of variables from the boundary condition on the impedance plane

$$
\left(\frac{\partial}{\partial y}+k \alpha\right) H^{\mathrm{in}}(\vec{r})=0, \quad y=-c .
$$

To ensure the solution uniqueness, the total field has to satisfy the 2-D Helmholtz equation, the boundary conditions on $M$ and impedance plane, the Meixner condition at the reflector edges, and the Sommerfeld radiation condition. In the case of lossless earth $(Z=-i|Z|)$ the latter condition must be modified according to [10], [11] in order to account for the surface-wave excitation. The scattered field can be presented in integral form as a double-layer potential over $M$

$$
H^{s c}(\vec{r})=\int_{M} \mu\left(\vec{r}_{s}\right) \frac{\partial}{\partial n_{s}} G\left(\vec{r}, \vec{r}_{s}\right) d l_{s}
$$


where

$\mu \quad$ unknown surface current density that we are seeking;

$G \quad$ "magnetic" Green's function of the half space bounded by the impedance plane;

$l_{s} \quad$ coordinate along the contour $M$.

The function $G$ can be derived explicitly in the Fourier transform domain. The final expression is very similar to (2)

$$
\begin{aligned}
G\left(\vec{r}, \vec{r}_{s}\right)= & \frac{i}{4} H_{0}^{(1)}\left(k\left|\vec{r}-\vec{r}_{s}\right|\right)+\frac{i}{4 \pi} \int_{-\infty}^{\infty} \frac{i g-\alpha}{g(i g+\alpha)} \\
& \cdot e^{i g k\left(y+y_{s}+2 c\right)+i h k\left(x-x_{s}\right)} d h
\end{aligned}
$$

however, here $\vec{r}_{s}$ is a real-valued source-coordinate vector. By imposing the boundary conditions on the PEC reflector, we arrive at the following EFIE:

$\frac{\partial}{\partial n} \int_{M} \mu\left(\vec{r}_{s}\right) \frac{\partial}{\partial n_{s}} G\left(\vec{r}, \overrightarrow{r_{s}}\right) d l_{s}=-\frac{\partial}{\partial n} H^{\text {in }}(\vec{r}), \quad \vec{r} \in M$

Equations of this type are often encountered in the 2-D wavescattering problems. Note that (6) is an integral equation of the first kind and its kernel is hypersingular. That is why a direct application of MoM for solving (6) cannot be backed by a proof of convergence and, hence, is not able to ensure a high accuracy of computations; besides, CPU time rapidly grows up for the larger reflectors due to either a need to invert a larger matrix or a larger matrix filling time. Instead, the solution scheme that is based on the analytical inversion of the static part of the free-space counterpart of (6) is much more efficient. This procedure is quite similar to the free-space and in-radome reflector analyses developed in [2], [7]; all the modifications are due to the presence of the second (Fourier integral) terms in (5) and (2). That is why here we only mention briefly the main points of the further derivations.

The surface current density $\mu\left(\varphi_{s}\right)$ is extended as identical zero beyond $M$ to the remaining part of the circle of the radius $a$. The discrete form of EFIE is obtained by expanding all the functions in (6) in terms of the entire-period angular exponents [2], e.g., $\mu\left(\varphi_{s}\right)=(2 / i \pi k a) \sum_{n=-\infty}^{\infty} \mu_{n} e^{i n \phi_{s}}$. Term-by-term integration and differentiation, together with the condition of a zero current off $M$, bring us to the dual series equations for the unknown expansion coefficients $\mu_{n}$

$$
\begin{aligned}
& \sum_{n=-\infty}^{\infty}\left[\mu_{n} J_{n}^{\prime}(k a)+J_{n}\left(k r_{c s}\right) e^{-i n \varphi_{c s}}\right] D_{n}\left(e^{i \varphi}\right) \\
& =0, \quad\left|\varphi-\varphi_{0}\right|<\theta_{a p} \\
& \sum_{n=-\infty}^{\infty} \mu_{n} e^{i n \varphi}=0, \quad \theta_{a p}<\left|\varphi-\varphi_{0}\right| \leq \pi
\end{aligned}
$$

where $J_{n}$ is the Bessel function and the prime denotes the derivative with respect to the argument. The coefficients in (7) are as follows:

$D_{n}\left(e^{i \varphi}\right)=H_{n}^{\prime(1)}(k a) e^{i n \varphi}+(-i)^{n} \sum_{p=-\infty}^{\infty} i^{p} J_{p}^{\prime}(k a) \Omega_{n+p} e^{i p \varphi}$ where

$$
\begin{aligned}
& r_{c s}=\sqrt{r_{0}^{2}-b^{2}+2 i b\left(x_{0} \cos \beta+y_{0} \sin \beta\right)} \\
& \varphi_{c s}=\arccos \left(\frac{x_{0}+i b \cos \beta}{r_{c s}}\right) \\
& \Omega_{n}=H_{n}^{(1)}(2 k c)-\frac{2 \alpha}{\pi} \int_{-\infty}^{\infty} \frac{(h-i g)^{n}}{g(i g+\alpha)} e^{2 i g k c} d h .
\end{aligned}
$$

Further, we extract out and invert analytically the static part of the free-space term (i.e., not containing $\Omega_{n}$ ) of the left-hand side of (7) and (8). In so doing we retrace all the steps of the freespace reflector scattering [2] except for the symmetry splitting of the surface current density, which is not possible here due to the presence of the earth. Finally, we arrive at the following infinite-matrix equation:

$$
\mu_{m}-\sum_{n=-\infty}^{\infty} A_{m n} \mu_{n}=B_{m}, \quad m=0, \pm 1, \pm 2, \cdots
$$

where

$$
\begin{aligned}
A_{m n}= & {\left[|n|+i \pi(k a)^{2} J_{n}^{\prime}(k a) H_{n}^{(1)^{\prime}}(k a)\right] T_{m n}+i \pi(k a)^{2} } \\
& \cdot(-i)^{n} J_{n}^{\prime}(k a) \sum_{p=-\infty}^{\infty} i^{p} J_{p}^{\prime}(k a) \Omega_{p+n} T_{m p} \\
B_{m}= & i \pi(k a)^{2} \sum_{n=-\infty}^{\infty} J_{n}\left(k r_{c s}\right) e^{-i n \varphi_{c s}} \\
& {\left[T_{m n} H_{n}^{(1)^{\prime}}(k a)+(-i)^{n} \sum_{p=-\infty}^{\infty} i^{p} J_{p}^{\prime}(k a) \Omega_{p+n} T_{m p}\right] . }
\end{aligned}
$$

The coefficients $T_{m n}$ can be found in [2], [7], and [10] and are easily computed as combinations of the exponents of $\varphi_{0}$ and the Legendre polynomials of $\cos \theta_{a p}$. Note that the first terms in (13), (14) coincide with the free-space reflector solution [2], while the second ones characterize the earth effect. Due to the fact that $\sum_{m, n=-\infty}^{\infty}\left|A_{m n}\right|^{2}<\infty$, the infinite-matrix equation (12) is a Fredholm second-kind one in the space $l_{2}: x \in l_{2}$ if $\sum_{n=-\infty}^{\infty}\left|x_{n}\right|^{2}<\infty$. This is ensured by the estimations valid for the matrix elements and the right-hand side vector components if $|m|,|n| \gg 1$. The estimates are derived in a straightforward but elaborate way and yield

$$
\begin{gathered}
\left|A_{m n}\right| \leq \frac{c_{1}}{|m|^{1 / 2}|n|^{3 / 2}|m-n+1|}+\frac{c_{2}}{|m|^{1 / 2} \ln |m|}\left(\frac{a}{c}\right)^{|n|} \\
\left|B_{m}\right| \leq \frac{c_{3}}{|m|^{3 / 2}} \sum_{n=0}^{\infty}\left[\left|\frac{r_{c s}}{a}\right|^{n}+\left|\frac{r_{c s}}{c}\right|^{n} \sum_{p=0}^{\infty}\left(\frac{a}{c}\right)^{p}\right]
\end{gathered}
$$

where $c_{1,2,3}$ are some constants. Hence, due to the Fredholm alternative and uniqueness theorem, exact solution to (12) exists in $l_{2}$ and can be approached to machine precision by solving progressively greater matrices [1]. Note, however, that (15) and (16) do not guarantee the convergence if $\left|r_{c s}\right| \geq a$ or $\left|r_{c s}\right| \geq c$ because of divergence of the series in the right-hand side of (16) and if $a \geq c$ due to divergence of (16) and the second term in (15). A more accurate estimation based on the properties of the functions $T_{m n}\left(\theta_{a p}\right)$ relaxes these conditions. It shows that the solution exists and numerical convergence takes place provided 
that the contour $M$, the source branch cut, and the earth interface do not intersect each other.

\section{Far-Field Characteristics and Power Conservation}

The far-field radiation pattern $\Phi^{\text {tot }}(\varphi)$ of our reflector antenna is obtained by applying the saddle-point technique to the evaluation of the integrals in (2) and (5). The result is

$$
\begin{aligned}
\Phi^{t o t}(\varphi)= & \Phi^{0}(\varphi)+\Phi^{s c}(\varphi) \\
\Phi^{0}(\varphi)= & e^{-i k\left(x_{0} \cos \varphi+y_{0} \sin \varphi\right)} \\
& {\left[e^{k b \cos (\varphi-\beta)}+\frac{i \sin \varphi-\alpha}{i \sin \varphi+\alpha} \cdot e^{2 i k\left(y_{0}+c\right) \sin \varphi} e^{k \cdot b \cos (\varphi+\beta)}\right] }
\end{aligned}
$$$$
\Phi^{s c}(\varphi)=\sum_{n=-\infty}^{\infty} \mu_{n}(-i)^{n} J_{n}^{\prime}(k a)
$$$$
\left(e^{i n \varphi}+\frac{i \sin \varphi-\alpha}{i \sin \varphi+\alpha} \cdot e^{2 i k c \sin \varphi-i n \varphi}\right) .
$$

Here, $\Phi^{0}(\varphi)$ is the full radiation pattern of the primary CSP feed in the presence of the impedance plane.

If $Z=-i|Z|$, then there are surface-wave poles on the real $h$-axis at $h= \pm h_{0}= \pm \sqrt{1+\alpha^{2}}$. To obtain the amplitudes of the corresponding guided modes at $x \rightarrow \pm \infty$ along the interface, one has to calculate the residues $B_{ \pm}$at the following poles:

$$
\begin{aligned}
B_{ \pm} & =B_{ \pm}^{0}+B_{ \pm}^{s c} \\
B_{ \pm}^{0} & = \pm \frac{4 \alpha}{h_{0}} e^{-\alpha k\left(y_{c s}+c\right)} e^{\mp i h_{0} k x_{c s}} \\
B_{ \pm}^{s c} & =\frac{4 \alpha}{h_{0}} e^{-\alpha k c} \sum_{n=-\infty}^{\infty} \mu_{n}(\mp i)^{n} J_{n}^{\prime}(k a)\left(h_{0} \pm \alpha\right)^{n} .
\end{aligned}
$$

The directivity $D$ in the main beam direction $\left(\varphi=\varphi_{0}\right)$ and the total radiated power $P_{\text {rad }}$ are obtained as, respectively,

$$
D=\frac{2 Z_{0}\left|\Phi^{t o t}\left(\varphi_{0}\right)\right|^{2}}{k P_{\text {rad }}}, \quad P_{\text {rad }}=\frac{Z_{0}}{\pi k} \int_{0}^{\pi}\left|\Phi^{\text {tot }}(\varphi)\right|^{2} d \varphi .
$$

Independent partial test of the numerical code can be performed by checking the power conservation law (also known as optical theorem [11], [12] in the plane wave scattering). In the case of a CSP beam incident on the reflector, one has to derive the modified expression of this sort. As the feed is a CSP one, with associated branch cut in real space, we choose a contour $C_{1}$ that coincides with the circle of radius $a$ and integrate the total outward power flow through this contour to evaluate the power coming from the feed $P_{\mathrm{in}}$. In order to calculate the total radiated power of the reflector antenna, we chose the infinitely remote contour $C \rightarrow \infty$ that completely encloses the reflector and can be divided into four parts in our geometry. Integration of the normal component of the Poynting vector along the semi-circular arc $C_{2}$ is reduced to the power radiated as a cylindrical wave along the contours $C_{3}$ and $C_{4}$ it is reduced to the powers $P_{s w \pm}$ of the right- and left-moving surface waves, respectively, and along the contour $C_{5}$ it represents the power absorbed in the lossy earth $P_{a b s}$. Depending on the electromagnetic properties of the earth, only one of the latter two power components will exist. Equivalently, the power-balance equation can be derived by applying the Green's formula [10], [11] to the total field function and its complex conjugate in the domain bounded by the mentioned closed contour, and then taking a limit of $C_{2}, C_{3}, C_{4} \rightarrow \infty$. On following either way, we find that

$$
P_{\mathrm{in}}=P_{r a d}+\left(P_{s w+}+P_{s w-}\right)+P_{a b s}
$$

where

$$
\begin{aligned}
P_{\mathrm{in}} & =-\frac{a Z_{0}}{2 k} \operatorname{Im}\left\{\left.\int_{0}^{2 \pi} H_{z}^{*} \frac{\partial H_{z}}{\partial r}\right|_{r=a} d \varphi\right\} \\
& =-\frac{2 Z_{0}}{k} \sum_{n=-\infty}^{\infty}\left\{\left|\xi_{n}\right|^{2}+R e\left[(-i)^{n} \xi_{n} \zeta_{n}^{*}\right]\right\} \\
\xi_{n} & =J_{n}\left(k r_{c s}\right) e^{-i n \varphi_{c s}}+\mu_{n} J_{n}^{\prime}(k a) \\
\zeta_{n} & =\sum_{m=-\infty}^{\infty} \xi_{m}(-i)^{m} \Omega_{n+m} \\
P_{s w \pm} & =(2 k \alpha)^{-1} Z_{0} h_{0}\left|B_{ \pm}\right|^{2}
\end{aligned}
$$

and (28), shown at the bottom of the page.

After a code verification, the power conservation law can be applied to avoid the numerical integrations for computing either $P_{a b s}$ or $P_{\text {rad }}$.

Although the earth impedance parameter $Z$ is usually small, its value influences noticeably on the wave radiation and propagation. Three basic cases can be considered:

1) $Z=0$ : a PEC plane which can be used as a model of the sea water interface. In this case, it is possible to avoid a time-consuming numerical integration to determine $\Omega_{n}$ as it reduces to $H_{n}^{(1)}(2 k c)$.

2) $Z=-i Y$ : a lossless impedance plane which can support a vertically polarized surface wave; it can be used as an idealized model of a thin ice layer on the metal roof or a thin dry sand layer on the wet substrate. Here $Y=$ $\epsilon^{-1 / 2} \tan \left(k h \epsilon^{1 / 2}\right)$, where $h$ and $\epsilon$ are the layer thickness and permittivity, respectively. In this case, the total far field consists of two parts: the field radiated in the upper half-space carrying the power $P_{\text {rad }}$ and the right- and leftmoving surface wave fields carrying the powers $P_{s w \pm}$.

3) $Z=X-i Y$ : a lossy impedance plane modeling the surface properties of arbitrary earth. In this case, the power absorbed in the earth $P_{a b s}$ should be calculated in addition to the radiated power $P_{\text {rad }}$.

$$
P_{a b s}=-\frac{Z_{0}}{2 k} \operatorname{Im}\left\{\left.\int_{-\infty}^{\infty} H_{z}^{*} \frac{\partial H_{z}}{\partial y}\right|_{y=-c} d x\right\}=\operatorname{Im} \alpha \frac{4 Z_{0}}{\pi k} \times \int_{-\infty}^{\infty}\left|\frac{e^{i g k c}}{i g+\alpha} \sum_{n=-\infty}^{\infty}(-i)^{n} \xi_{n}(h+i g)^{n}\right|^{2} d h .
$$



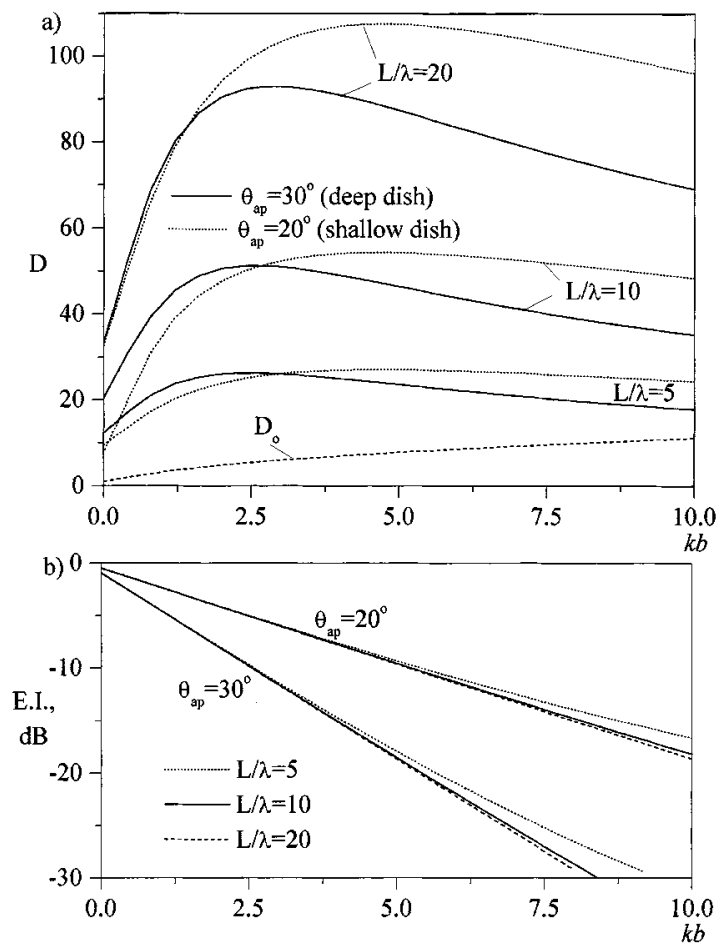

Fig. 2. (a) Free-space reflector directivity. (b) Edge illumination and as a function of the complex source-point parameter $k b$.

The effect of the surface wave or absorption losses on the antenna performance is characterized by the radiation efficiency $\eta$ and the directive gain $G_{d}$, respectively,

$$
\eta=\frac{P_{\text {rad }}}{P_{\text {rad }}+\left(P_{s w+}+P_{s w-}\right)+P_{a b s}}, \quad G_{d}=\eta D .
$$

It is only in the case of PEC earth and PEC reflector that the radiation efficiency is $100 \%$, so that the terms gain and directivity are equivalent to each other.

\section{NUMERICAL RESULTS AND DISCUSSION}

\section{A. Details of Computations}

In this section, we present and discuss the main antenna characteristics obtained for various reflectors, feed directivities, elevations above the earth, and earth properties. All the computations were performed by taking the matrix truncation number as $N_{t r}=k a+10$, which ensured the accuracy of $10^{-4}$ in calculating the radiation characteristics. The power conservation law (24) was always satisfied at the level of $10^{-12}$ as the double-precision computations were used; this is one of the merits of MAR. Cylindrical functions, including those of complex argument, were computed by a recursive code published in [13]. Special attention must be paid to the numerical integration in $\Omega_{n}$ functions. Here, after reducing the integration interval to a semi-infinite one, the latter was shifted down on a finite interval from the real $h$-axis in the complex plane to bypass the branch point at $h=1$ and a possible pole at $h=h_{0}$. As the elevation parameter $k c$ was quite large, this shift had to be of a small value in the order of $1 / k c$ to avoid a loss in the integration accuracy because of the exponential growth of the integrand in (11). Integration
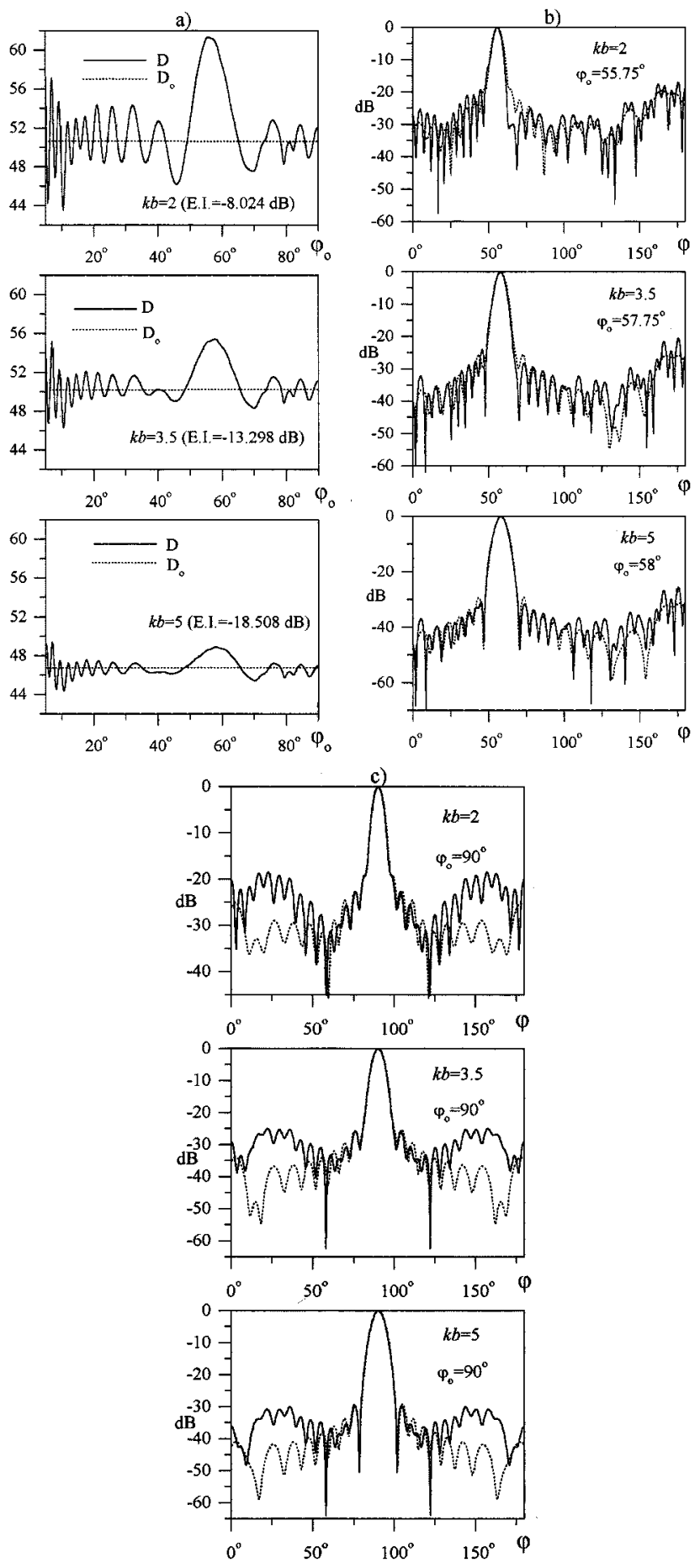

Fig. 3. (a) Directivity as a function of the reflector antenna aiming angle above the horizon of PEC earth for three values of edge illumination by a GO-focus feed; reflector parameters: $k a=62.8$ and $\theta_{a p}=30^{\circ}(L=10 \lambda, F / L=$ $0.5)$ and (b) and (c) normalized far-field patterns at the same values of edge illumination as in (a) for the angles of maximum directivity and "in zenith," respectively. Free-space comparison values are given by the dashed curves.

can be terminated at $15 / k c$ due to exponential decrement of the integrand along the real $h$-axis.

Computations were performed with a Pentium 100 platform and MS-DOS Fortran; typical CPU time was $85 \mathrm{~s}$ for $k a=62.8$, $a / c=1.01$, and $Z=0.23-i 0.01$, independently of $k b, \theta_{a p}$ and $\varphi_{0}$. 

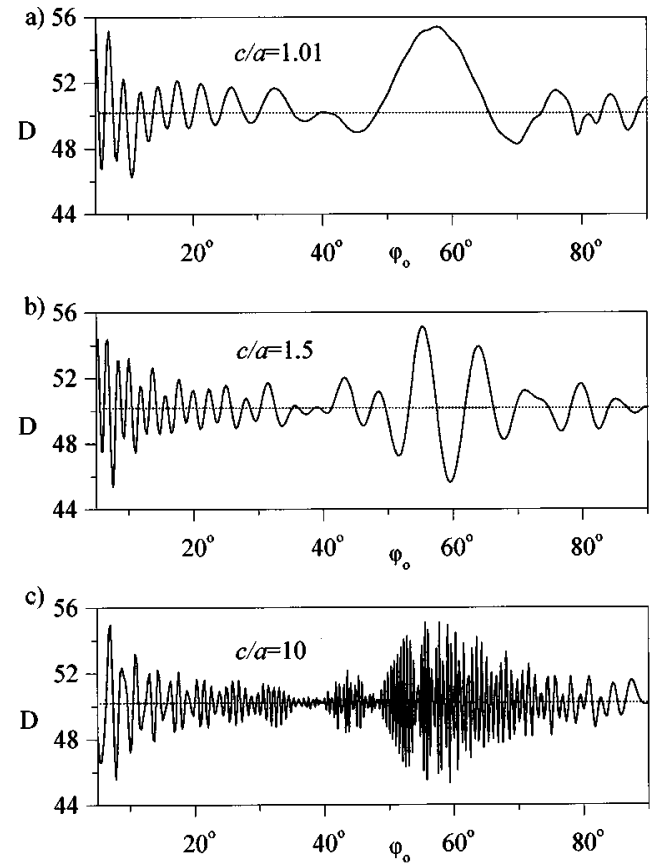

Fig. 4. Directivity of the same reflector near PEC earth as in Fig. 3 as a function of the aiming angle for three different elevations above the earth.

\section{B. Characterization of the Radiation Field}

For a device that has many parameters influencing its performance, it is advisable to select first a few primary parameters that have a greater effect than the others. In the reflector antenna case, the edge illumination is clearly one of such principal parameters. That is why first of all we studied how the antenna directivity depends on the directivity of the feed in the absence of the earth. It may seem that the increase of the feed directivity will always increase the directivity of the antenna, since the diffraction effect is reduced. However, an accurate numerical analysis shows that the directivity versus $k b$ has a broad maximum at a rather small value of $k b$. These plots are given in Fig. 2(a) for the various ratios of $L / \lambda, L$ being the aperture dimension and two values of $\theta_{a p}$ corresponding to $F / L=0.5$ and 0.73 . Therefore, to obtain the best antenna directivity one should first choose the appropriate value of the feed directivity or equivalently, the level of the edge illumination as depicted in Fig. 2(b). ${ }^{1}$ It is clearly visible that the edge illumination at approximately $-10-\mathrm{dB}$ level provides the maximum free-space directivity of a reflector.

For the rest of computations, we have chosen one basic reflector and feed geometry in order to concentrate our analysis on the effect of earth. This was a $L=10 \lambda, F / L=0.5$ reflector $\left(k a=62.8, \theta_{a p}=30^{\circ}\right)$ symmetrically fed by CSP feed placed in GO focus. The deviation of such a reflector from a parabola is less than $0.09 \lambda$ [2]. The plots in Fig. 3(a) present the dependence of directivity on the antenna aiming angle above the horizon for three values of the feed directivity; the edge illumination level is indicated in brackets. For simplicity, we considered here an antenna over the PEC earth: $Z=0$. The dashed line corresponds

\footnotetext{
${ }^{1}$ There is a misprint in the legend to the similar plots in [2, fig. 10]: they correspond to $k a=121.4$ and $\theta_{a p}=15^{\circ}$; besides, the correct values of $D$ are twice smaller than given in that figure.
}

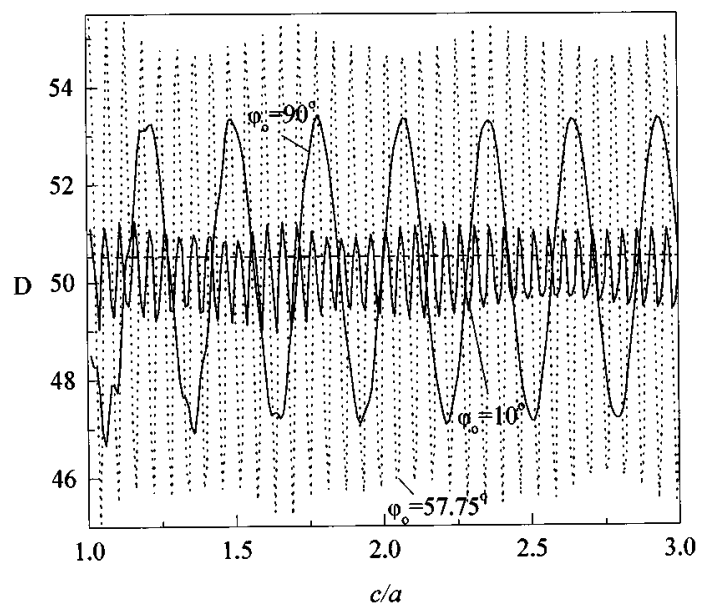

Fig. 5. Directivity of the same reflector near PEC earth as in Fig. 3 as a function of the normalized elevation, for in-zenith and near-grazing radiation.
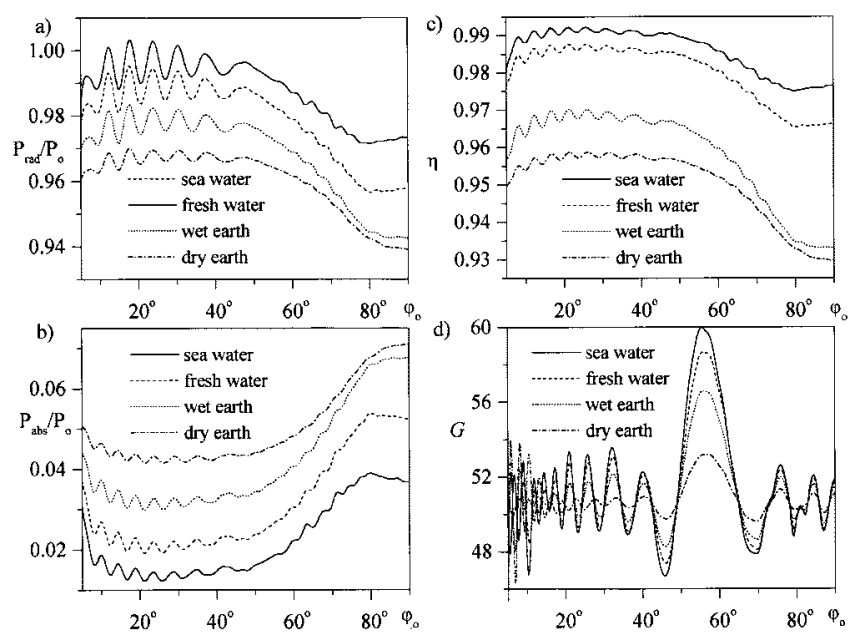

Fig. 6. (a) Normalized radiated power, (b) absorbed power, (c) radiation efficiency, and (d) antenna gain as a function of the aiming angle, for the same reflector as in Fig. 3 but near four types of lossy earth.

to the free-space directivity $D_{0}$ of the same antenna. One can see that the directivity value is oscillating near $D_{0}$; these oscillations are greater in the case of stronger edge illumination. The angle of the maximum directivity is easily identified as that for which one of the spillover sidelobes is reflected from the earth at the same angle as antenna's main beam (near to $60^{\circ}$ in our example). In Fig. 3(b) and (c), the radiation patterns are shown for the antennas inclined at the angle of the maximum directivity, and for those looking upwards at $\varphi_{0}=90^{\circ}$, respectively; free-space forward radiation patterns are added as dashed curves. One can notice that the pattern distortions are greater in the latter case, as here the spillover radiation around the both edges of reflector experiences a reflection from the earth.

Elevation above the earth is another factor affecting the radiating characteristics of reflector antenna. Plots in Fig. 4 show that with increasing the distance between the earth and the antenna, the directivity as a function of the aiming angle $\varphi_{0}$ does not change its basic behavior but the oscillations become denser. This is natural as the reason of oscillations is the interference between the main beam and the edge-diffracted and further earth- 
TABLE I

FOUR TYPES OF LOSSY SURFACES ON THE ANTENNA RADIATION

\begin{tabular}{l||l|l|l}
\hline & $\epsilon$ & $\sigma, S / m$ & \multicolumn{1}{c}{$Z$} \\
\hline sea water & 80 & 1 & $0.0597-i 0.0339$ \\
\hline fresh water & 80 & $10^{-3}$ & $0.1118-i 1.258 \cdot 10^{-4}$ \\
\hline wet earth & 20 & $10^{-2}$ & $0.229-i 0.01$ \\
\hline dry earth or sand & 4 & $10^{-3}$ & $0.5-i 1.123 \cdot 10^{-2}$ \\
\hline
\end{tabular}

a)
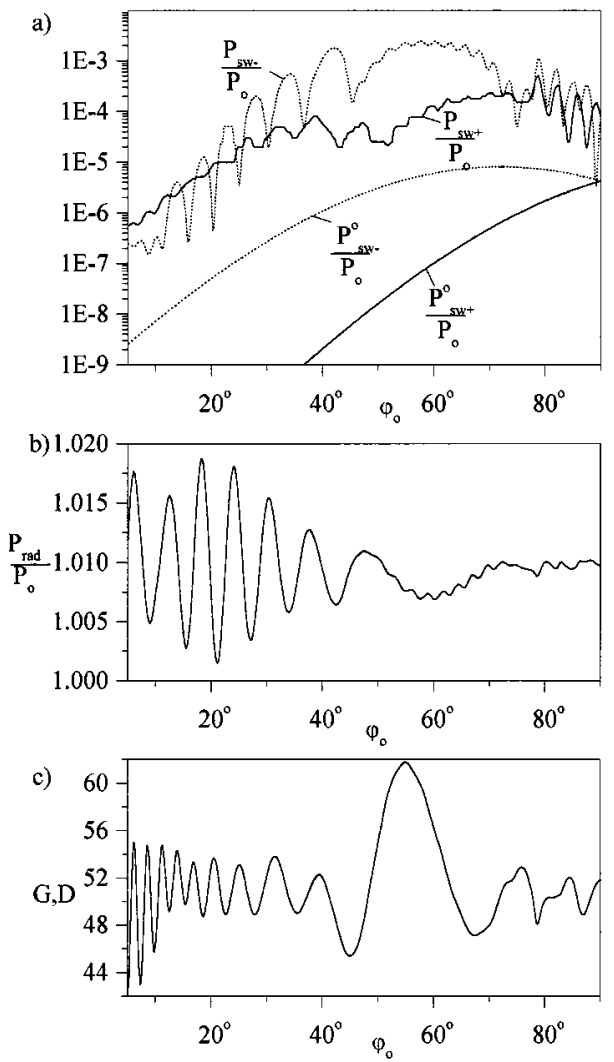

Fig. 7. (a) Normalized radiated power, (b) surface wave power, and (c) antenna gain and directivity as a function of the aiming angle for the same reflector as in Fig. 3, but near PEC earth covered with a dielectric layer.

reflected radiation. The same relates to the plots in Fig. 5 presenting the directivity versus the normalized distance $c / a$ between the earth and the center of reflector curvature.

Fig. 6 compares the effect of the four types of lossy surfaces on the antenna radiation shown in Table I.

All the power characteristics are normalized to the radiation power of the same CSP feed (2) located in the free space: $P_{0}=$ $2 Z_{0} k^{-1} I_{0}(2 k b)$, where $I_{0}$ is for the modified Bessel function (see [2]). This implies that we assume the virtual current in the CSP feed be the same in two situations. The presence of the flat earth does not have a strong effect on the radiated power [Fig. 6(a)]. The absorbed power [Fig. 6(b)] is small, but it increases for the larger aiming angles $\varphi_{0}$ because then the both edge-spillover sidelobes hit the earth. This results in a lower radiation efficiency $\eta$ of a zenith-looking antenna (Fig. 6(c)). The gain of antenna $G_{d}$, which is equal to $\eta D$, can vary within $10 \%$ due to the earth properties depending on the inclination as in Fig. 6(d); this means that such an effect may take place within one day due to the drying of wet earth around a reflector.
For an antenna located over a PEC metal roof with a thin lossless ice layer of $\epsilon=2.5$ and $k h=0.194$, the normalized power fractions are shown in Fig. 7(a) and (b). This situation corresponds to the impedance value $Z=-i 0.2$. Here $P_{s w \pm}$ and $P_{s w \pm}^{0}$ are the right (left) moving surface wave powers with and without the reflector, respectively. One may clearly see the role played by the edge diffraction in the enhanced surface wave excitation. However, general level of the surface wave losses is quite low, so that the gain and directivity plots in Fig. 7(c) are indistinguishable.

\section{CONCLUSIONS}

The complex source-point approach combined with MAR has been used to analyze a circular cylindrical antenna over the imperfect earth. Resulting Fredholm second-kind matrix equation for the surface-current expansion coefficients provides a convergent numerical solution. Various antenna characteristics have been calculated and the influence of the earth properties on the antenna radiation has been studied. There are a number of radiation features not predicted by approximate methods, e.g., the sidelobe level and, hence, the directivity can be both lower and higher than in the free-space depending on the antenna aiming angle and elevation. In the directivity values of the same antenna over sea and dry earth, the difference can be as great as $10 \%$ of the peak value, etc. A practical application of these conclusions can be seen in the following suggestion: if reflector antenna operates with a fixed aiming angle, its directivity can be improved simply: 1) by adjusting the plane of the local "earth" to reflect the spillover sidelobe in the main beam direction and 2) by making the "earth" (e.g., a wall) of metallic material. Note that the guaranteed accuracy of the computation results makes a numerical optimization of antenna geometry possible.

If a reflector is not circular, a similar approach can be developed, but the matrix elements then require additional terms to be found by numerical integration. Although we have considered a reflector near the impedance plane, the method is applicable to various layered media; one needs only to determine first the Green's function of the corresponding host medium. Besides, it is easy to apply the same technique for the analysis of a subsurface radar or an antenna submerged in water. MAR approach can be used to simulate 3-D spherical reflector antennas in the layered media as well, but in this case the regularization procedure is based on another analytical technique [3].

\section{ACKNOWLEDGMENT}

The authors would like to thank V. B. Yurchenko for discussions. A. I. Nosich would like to thank Politecnico di Torino for the hospitality during his stay there as a NATO-CNR Guest Fellow.

\section{REFERENCES}

[1] A. I. Nosich, "The method of analytical regularization in wave scattering and eigenvalue problems: Foundations and review of solutions," IEEE Antennas Propagat. Mag., vol. 42, pp. 34-49, June 1999.

[2] T. Oğuzer, A. Altintaş, and A. I. Nosich, "Accurate simulation of reflector antennas by the complex source-dual series approach," IEEE Trans. Antennas Propagat., vol. 43, pp. 793-801, Aug. 1995. 
[3] S. S. Vinogradov, P. D. Smith, E. D. Vinogradova, and A. I. Nosich, "Accurate simulation of a spherical reflector front-fed by a Huygens CSP beam: A dual series approach," in Proc. Int. Symp. Antennas JINA, Nice, France, Nov. 1998, pp. 550-553.

[4] L. B. Felsen, "Complex-point source solution of the field equations and their relation to the propagation and scattering of Gaussian beams," Symp. Math., vol. 18, pp. 39-56, 1975.

[5] E. V. Jull and G. A. Suedan, "Beam diffraction by planar and parabolic reflectors," IEEE Trans. Antennas Propagat., vol. 39, pp. 521-527, Apr. 1991.

[6] A. I. Nosich, V. B. Yurchenko, and A. Altintaş, "Numerically exact analysis of a 2-D variable-resistivity reflector fed by a CSP," IEEE Trans. Antennas Propagat., vol. 45, pp. 1592-1601, Nov. 1997.

[7] V. B. Yurchenko, A. Altintaş, and A. I. Nosich, "Numerical optimization of a cylindrical reflector-in-radome antenna system," IEEE Trans. Antennas Propagat., vol. 47, pp. 668-673, Apr. 1999.

[8] L. B. Felsen and N. Marcuvitz, Radiation and Scattering of Waves. Englewood, NJ: Prentice-Hall, 1973.

[9] G. S. Smith, "Directive properties of antennas for transmission into a material half-space," IEEE Trans. Antennas Propagat., vol. AP-32, pp. 232-246, Mar. 1984.

[10] A. I. Nosich, "Green's function-Dual series approach in wave scattering by combined resonant scatterers," in Analytical and Numerical Methods in Electromagnetic Wave Theory, M. Hashimoto, M. Idemen, and O. A. Tretyakov, Eds. Tokyo, Japan: Science House, 1993, pp. 419-469.

[11] A. I. Nosich, "Radiation conditions, limiting absorption principle and general relations in open waveguide scattering," J. Electromagn. Waves Applicat., vol. 8, no. 3, pp. 329-353, 1994.

[12] P. G. Petropoulos and G. A. Kriegsmann, "Optical theorems for electromagnetic scattering by inhomogeneities in layered dielectric media," IEEE Trans. Antennas Propagat., vol. 39, pp. 1119-1124, Aug. 1991.

[13] C. F. du Toit, "Evaluation of some algorithms and programs for the computation of integer-order Bessel functions of the first and second kind with complex arguments," IEEE Antennas Propagat. Mag., vol. 35, pp. 19-25, June 1993.

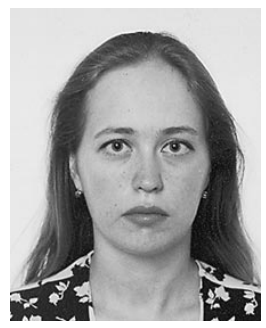

Svetlana V. Boriskina (S'96) was born in Kharkov, Ukraine, in 1973. She received the M.S. and Ph.D. degrees from Kharkov State University, Ukraine, in 1995 and 1999, respectively.

She is currently working as a Researcher at the Radiophysics Department, Kharkov State University. Her research interests are in integral equation methods and electromagnetic wave scattering from dielectric and metallic scatterers in layered media, with applications to open waveguides and antennas.

Dr. Boriskina received the Graduate Fellowship in Advanced Electromagnetics of the SUMMA Foundation in 1996 and the IEEE Microwave Theory and Technique Society Graduate Student Scholarship Award in 1997.

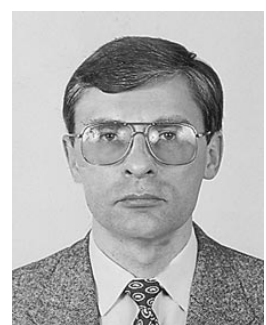

Alexander I. Nosich (M'94-SM'95) was born in Kharkov, Ukraine, in 1953. He received the M.S., $\mathrm{Ph} . \mathrm{D}$., and D.Sc. degrees in radio physics from the Radio Physics Department, Kharkov State University, in 1975, 1979, and 1990, respectively.

Since 1978, he has been on the research staff of the Institute of Radiophysics and Electronics (IRE), Ukrainian Academy of Sciences, Kharkov. From 1992 to 1998 he held research fellowships and visiting professorships in Bilkent University, Ankara, Turkey; Kumamoto University, , Gifu University, , and Chuo University, Tokyo, Japan; University of Rennes 1, France; and Torino Polytechnical University, Italy. Currently, he is with IRE, Kharkov, as a leading scientist in the Department of Computational Electromagnetics. Since 1995 he has been on the editorial board of Microwave and Optical Technology Letters. His research interests include free-space and open-waveguide scattering, complex-mode behavior, radar cross-section analysis, and antenna simulation.

Dr. Nosich was the organizer and chairman of the IEEE Antennas and Propagation Society East Ukraine Chapter 1995, the first one in the former Soviet Union. From 1990 to 1998 he was the organizer and Technical Program Committee Chairman of a series of international conferences on Mathematical Methods in Electromagnetic Theory (MMET) held in Ukraine.

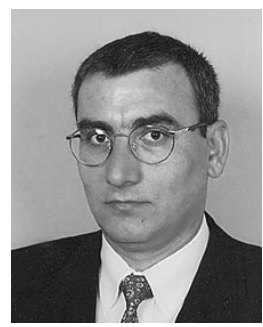

Ayhan Altintaş (S'82-M'86-SM'93) was born in Yozgat, Turkey, on March 29, 1958. He received the B.S. and M.S. degrees from the Middle East Technical University (METU), Ankara, Turkey, in 1979 and 1981, respectively, and the Ph.D. degree from the Ohio State University, Columbus, in 1986, all in electrical engineering.

From 1981 to 1987, he was with the Electro-Science Laboratory, Ohio State University. At present, he is a Professor at the Department of Electrical and Electronic Engineering, Bilkent University, Ankara, Turkey. He has held research fellowship and Guest Professor positions at Australian National University, Canberra, Australia, Tokyo Institute of Technology, Tokyo, Japan, and Technical University of Munich, Germany. His research interests include electromagnetic radiation and scattering, microwaves, fiber optics, and integrated optics.

Dr. Altintas received the ElectroScience Laboratory Outstanding Dissertation Award in 1986, the IEEE 1991 Outstanding Student Branch Counselor Award, the 1991 Research Award of the Professor Mustafa N. Parlar Foundation of METU, and the 1996 Young Scientist Award of TUBITAK. He is a member of Sigma Xi and Phi Kappa Phi. 